\title{
Computational Creativity
}

\author{
Włodzisław Duch Senior Member, IEEE
}

\begin{abstract}
Creative thinking is one of the hallmarks of human-level competence. Although it is still a poorly understood subject speculative ideas about brain processes involved in creative thinking may be implemented in computational models. A review of different approaches to creativity, insight and intuition is presented. Two factors are essential for creativity: imagination and selection or filtering. Imagination should be constrained by experience, while filtering in the case of creative use of words may be based on semantic and phonological associations. Analysis of brain processes involved in invention of new words leads to practical algorithms that create many interesting and novel names associated with a set of keywords.
\end{abstract}

\section{INTRODUCTION}

Human-level intelligence is in many respects far beyond what computational intelligence may provide now. Traditionally cognitive psychology divides human cognitive faculties into low-level and high-level. The low-level cognitive functions involve perception and motor control; most of these functions are not more developed in humans than in mammals and other animals. The high-level functions, involving thinking, reasoning, planning and language, are in most part unique to humans. This division is of course only approximate, but surprisingly it is mirrored by the computational intelligence community, divided between two large camps: pattern recognition using neural networks and other techniques that learn from data, focused on problems related to perception, object recognition and memory, and artificial intelligence focused on symbolic knowledge engineering, logic, theorem proving, or linguistic problems. Cognitive robotics is a natural meeting ground for these two communities because it requires integration of low and highlevel cognition in one system.

Creativity is still rather neglected subject. MIT Encyclopedia of Cognitive Sciences has about 1100 pages but devotes only a single page to creativity, and another page to creative person. Intuition is not mentioned in its index at all. In contrast to creativity and intuition 6 articles are devoted to logic, and logic is mentioned in the index almost 100 times. Yet it may be argued that mental acts are rarely based on logic, or well approximated using logic, but frequently on intuitive and creative thinking. Creativity, defined by Sternberg [1] as "the capacity to create a solution that is both novel and appropriate", manifests itself not only in creation of novel theories or inventions, but permeates our everyday actions, understanding of language and interactions among people. On the road towards systems with human-level

Włodzisław Duch is with the School of Computer Engineering, Nanyang Technological University, Signapore, and Nicolaus Copernicus University, Torun, Poland (Google: Duch, or www.phys.uni.torun.pl/ duch) competence understanding and implementation of creativity in computational systems should be of central concern.

Despite the limitation of the current knowledge of the neural processes that give rise to the higher cognitive processes in the brain it is possible to propose a testable, neurocognitive model of creative processes. A brief review of the psychological perspectives and neuroscientific experiments on creativity is presented in the next section. This is followed by a general description of putative brain processes that lead to creativity. This model is then simplified and applied to one of the simplest domains where creativity is manifested: invention of novel, interesting words. Discussion of future direction of the neurocognitive approach to creativity closes this paper.

\section{CREATIVITY FROM PSYCHOLOGICAL AND NEUROSCIENTIFIC PERSPECTIVE}

Creativity is manifested in many areas, including invention of new concepts and ideas or production of objects of art and music. The degree of creativity involved in different activities depends on the cultural context at a given point in time. Bink and Marsh write that "there are as many researchbased definitions of creativity as there are approaches to studying the topic" [2]. Boden [3] writes that creativity is "a matter of using ones computational resources to explore, and sometimes to break out of, familiar conceptual spaces." She distinguishes two dimensions of creativity: historical creativity (H-creativity) vs. personal, or psychological creativity (P-creativity), when the idea is new for the humanity or only for the agent (person) generating the idea; and exploratory creativity (E-creativity) vs. transformational creativity (Tcreativity), based either on exploration of conceptual spaces appropriate for the task, or allowing for a change of the rules that are used to define conceptual spaces. Exploratory creativity would then be more incremental and combinatorial in nature, while transformational creativity would be a deeper form, perhaps even a paradigm shift.

Creativity research lies mostly in the domain of philosophers, educators and psychologists, with research reports appearing in two specialized journals: Creativity Research Journal and Journal of Creative Behavior. J.P. Guilford [4] introduced tests to measure fluency, flexibility, and originality of thought, still used in both visual and verbal domains. Sternberg has edited the "Handbook of Human Creativity" [1]. In his theory of successful intelligence [5] he distinguished between creative intelligence and cognitive intelligence claiming that the former is more sensitive to individual and developmental differences than the latter. The history and current state of research on the mathematical creativity is presented in [6], and psychological review articles in [7]. 
All aspects of creativity research were collected very recently in the "Encyclopedia of creativity" [8], written by 167 experts. Only a few articles in this Encyclopedia (by Pribram, Proctor and Schuldberg) concern general brain processes, chaos and dynamical systems, but no testable neurological or computational models of creativity have been proposed.

Intelligence is frequently reduced to a single, measurable G-factor because the Intelligence Quotient test results are positively correlated with scores of many independent tests of quite diverse cognitive abilities [9]. Nevertheless Gardner's idea of multiple intelligences is now commonly accepted [10]. Linguistic and logical-mathematical forms of intelligence are measured in IQ tests, but musical, artistic, spatial, and bodily-kinesthetic intelligences are of a different kind. Interpersonal intelligence, including emotional intelligence, is also rather different and not necessarily correlated with other types of intelligence. In all these areas one can exhibit intelligent, creative behavior. Different aspects of intelligent behavior analyzed from psychological perspective may result either from diffuse recruitment of multiple brain regions that cooperate in an efficient way to solve the task, or from a specific system that controls diverse forms of behavior. Brain imaging studies [11] show that such central executive is indeed involved, and intelligence in its many forms depends on the working memory.

Computational models need as many constraints coming from neuroscience as possible. Unfortunately direct brain imaging observations of creative thinking or action have not been done yet. The "Aha!" or Eureka phenomenon, an insight experience [12] during problem solving, has been studied using functional magnetic resonance imaging (fMRI) and electroencephalography (EEG) techniques, contrasting observations with analytical problem solving that did not required insight [13]. After solving a problem presented in a verbal way subjects indicated themselves whether they had an insight or not. An increased activity of the right hemisphere anterior superior temporal gyrus was observed during initial solving efforts and during insights. About $300 \mathrm{~ms}$ before insight a burst of gamma EEG activity was observed. This has been interpreted by the authors as "making connections across distantly related information during comprehension ... that allow them to see connections that previously eluded them" [13]. This process has been investigated by Bowden and collaborators [14], who imagine that initial impasse is due to the inability of left hemisphere, focused on the problem, to make progress, and that it is overcome when lessfocused right hemisphere adds relevant information while strong activation diminishes with time, allowing other associations to be formed. In their view subjective Aha! experience results from activation by the pre-existing weak solution in the right hemisphere suddenly reaching consciousness when the overshadowing activation diminishes. Experiments conducted by Bowden et al. showed that fMRI activations for insight versus non-insight problem-solving were localized in the right-hemisphere anterior superior temporal gyrus (RH-aSTG). This area is probably involved in higher-level abstractions that can facilitate indirect associations, and one should expect that the same neural structures should be involved in creative thinking.

Intuition is also a concept difficult to grasp, but commonly believed to play an important role in business and other decision making. Sinclair and Ashkanasy [15] define intuition as "a non-sequential information-processing mode, which comprises both cognitive and affective elements and results in direct knowing without any use of conscious reasoning". It has also been defined as "knowing without being able to explain how we know" [16], [17]. Tests to measure intuition were introduced by Wescott already in 1961 . Intuition probably relies on implicit learning, gaining tacit knowledge without being aware of learning. Psychologists advance our understanding of the concept and measurement of intuition comparing responses to different types of tests and inventories, such as the Myers-Briggs Type Inventory (MBTI) or the Accumulated Clues Task (ACT). Different intuition measures are not correlated, showing problems in constructing theoretical concept of intuition [18]. Direct attempt to study relationships between intuition and creativity has been reported in [19]. Significant correlations were found between the Rational-Experiential Inventory (REI) intuition scale and some measures of creativity. Lieberman [20] argues that social intuition (which is the basis of nonverbal communication) is a phenomenological and behavioral correlate of knowledge gained through implicit learning. Intuition does not involve insight into structural relations, only judgment or fast behavioral response capable of accurately extracting probabilistic contingencies. He also reviews the neurobiological and neuropsychological evidence that the caudate and putamen structures of basal ganglia are involved in both implicit learning and intuition.

Mathematical intuition is an interplay between spatial imagination, abstraction and approximate reasoning, and analytical reasoning. This requires an interaction between visual-spatial and linguistic thinking, and has indeed been observed in brain imaging studies [21]. Analysis of Ramanujan's intuitive approach to mathematics and its relation to conventional cognitive processes may be found in Section 4.3 of [22]. It seems quite reasonable that distributed processes employed in intuitive thinking (Ramanujan was not able to justify his results) based on self-taught techniques go a long way towards explanation of genius traits.

The original four-stage Gestalt model of problem solving consists of preparation, incubation, illumination and verification steps. These stages were identified in creative problem solving by individuals and small groups of people [23]. Additional stages may involve elaboration on the preparation stage that is preceded by finding or noticing a problem and proposing interesting questions, frustration period that may precede illumination, and the final stage of communication that follows the verification stage. These stages do not have to be sequential, may be revisited several times, and may manifest in a different way in artistic, literary, scientific or management activities. Lubart concludes [23] that under- 
standing more details about various subprocesses and the way they are sequenced yielding creative productions is a central issue for creativity research.

Relationships between creativity and associative memory processes have been analyzed using pairwise word association technique in [24]. Semantic or phonological (spelling) priming was applied, showing for a brief (200 ms) moment an additional word before the second word of the pair was displayed. Creativity was positively correlated with greater ability to associate words and with susceptibility to priming, with distal associations showing longer latencies before making decision. Less creative people were not able to make such associations. Neutral priming, based on the nonsensical or unrelated words, also increased the number of claims that words are related. These results support the idea that creativity relies on the associative memory, and in particular on the ability to link together distant concepts. Priming with nonsensical sounds partially activates many words facilitating associations. Some puzzling results of this experiment are addressed in section 4 .

Psychological tests indicate that brains of creative people accept more incoming stimuli from the surrounding environment [25]. Creative individuals have low levels of latent inhibition responsible for filtering stimuli that seem to be irrelevant on the basis of past experiences. This may probably be related to more complex representation of objects and situations in creative minds. The need for everyday creativity has been almost completely neglected in the artificial intelligence research and may be credited for failures of some AI programs.

Analysis of creative ideas used in advertising [26] and product innovation [27] demonstrated that anarchist type of methods encouraging unstructured approach (free associations, brainstorming, random stimulation or lateral thinking) usually fail, while structured approaches, based on higherorder rules and templates, lead to excellent results. For example, $25 \%$ of the award-winning advertisements were based on a single "Replacement" template, while $89 \%$ of ads could be generated by one of the six "creativity templates." This process may be presented in an algorithmic way, with finite number of symbols and operators, and used to suggest new advertising ideas. Computer generated ideas based on templates were rated significantly higher both for creativity and originality than the ideas of non-professional people working without any constraints.

Creativity is difficult to investigate because of its seemingly fluid character, connections with poorly understood phenomena such as intuition, insight and genius studies, as well as philosophical and psychological pre-scientific bias (for example, Bergson or Jung - for a detailed review see [28]), and dependence on social context. Richards (in [8]) emphasizes creativity in everyday activities, providing flexible solutions to everyday problems. This is supported by Bink and Marsh [2] who stress that creative processes are based on the same cognitive processes as mundane thinking. The "Geneplore" model of creative behavior [29], well known in design, assumes interaction of generative processes and exploratory processes. Preinventive cognitive structures representing novel object forms, mental models, or verbal combinations, are generated, refined and regenerated to create mental representations.

\section{CREATIVITY FROM COMPUTATIONAL PERSPECTIVE}

Psychological and neuroscientific literature strongly argues that creativity is a product of ordinary cognitive processes and as such should be amenable to computational modeling. However, the lack of understanding what exactly is involved in creative activity is one of the main reasons for the low interest of the computational intelligence community in creative computing. Although no direct attempt to capture creative brain-like processes has been made so far, work in several directions is worth noting.

First, creativity is usually associated with discovery of new knowledge and therefore work on computer approaches that automatically discover new knowledge is relevant here. Langley, Simon, Bradshaw and Żytkow proposed a heuristic search-based AI models for modeling creative processes that led to historical scientific discoveries [30]. This led to the development of the "discovery science", focused on understanding historical discoveries of numeric and qualitative laws, as well as search for new scientific knowledge. Application to astronomy, elementary particle physics, superconductivity, chemistry and biology, generated some interesting models [30], [31], [32], although quite different from models generated by experts. There were no attempts to connect search-based approaches to processes in the brain.

Research in automatic programming goes in similar direction. Koza et al. [33] has used genetic programming as an automated invention machine in automated synthesis of antennas, analog electrical circuits, controllers, metabolic pathways (networks of chemical reactions), genetic networks and other areas, creating many useful patentable inventions. In most cases these inventions are optimized versions of known designs, therefore they should be considered rather as a routine improvements than truly creative inventions.

The most interesting computational models directly addressing creativity are Copycat, Metacat, and Magnificat models developed in Hofstadter's group [34], [35], [22]. These models define and explore "fluid concepts", that is concepts that are sufficiently flexible and context-sensitive to lead to automatic creative outcomes in challenging domains, such as the artistic design of letters in the Letter Spirit project [22]. Starting with examples of letters in a new style the program was able to design the remaining letters in similar style. Copycat architecture is based on an interplay between conceptual and perceptual activities. The first is implemented in a Slipnet spreading activation network, playing the role of the long-term memory, storing concepts, from simple objects to abstract relations. Links have length that reflect the strength of relationships between concepts, and change dynamically under the influence of the Workspace network, representing perceptual activity in the working memory. Numerous software agents, randomly 
chosen from a larger population, operate in the Workspace, assembling and destroying structures on various levels. The Copycat architecture estimates "satisfaction" derived from the content of assembled structures and concepts. Thus relations (and therefore the meaning) of concepts and highlevel perceptions emerge as a result of a large numbers of parallel, low-level, non-deterministic elementary processes. This indeed may capture some fundamental processes of creative intelligence, although connections with real brain processes are not discussed [22], [34], [35].

\section{NEUROCOGNITIVE APPROACH TO CREATIVE AND INTUITIVE COMPUTING}

The only truly intelligent devices that can solve all kind of complex problems are human brains. Cognitive science research led to interesting architectures based on formal models of cognition that do not seem to scale up to real world problems. Neurocognitive approach to intuition and creativity advocated here is based on inspirations from putative brain processes responsible for normal cognitive functions. From computational perspective logic and comprehensible sets of logical rules expressed in symbolic language may be used only for simple categorization and reasoning [36]. More complex intuitive categorization based on similarity to previously observed cases can sometimes be justified using fuzzy rules [37]. Threshold logic rules implemented by neural systems may have more expressive power [38]. A model of intuitive problem solving with concepts defined by probability density distributions over combinations of feature values in psychological spaces has been presented in [39], [40].

To simplify the large-scale neurodynamics of the brain one should focus on resonant states between microcircuits that belong to different minicolumns, as Grossberg has done in the Adaptive Resonance Theory (ART) [41]. Memory traces are coded by attractor states binding together activity of many microcircuits that represent elementary features derived from sensory inputs or hierarchically build upon combinations of such features. In some circumstances all modes of a complex dynamical system may be controlled (enslaved) by only a few modes. Attractors of such systems may lie on a low-dimensional hyperplane in the state space of a huge number of dimensions [42]. Some aspects of the world may become internalized in "neural spaces" [43]. It is probable that all mind events, including learning, take place in relatively low dimensional spaces that may be identified with "psychological spaces", or "conceptual spaces" [40]. Mental phenomena may be discussed in more natural way using language of psychological spaces rather than language of neurophysiological states. Seeing an object or hearing a sound is reflected in brain dynamics, evoking mental objects. This process may be modeled as activation of points in psychological spaces, belonging to a complex fuzzy region that represents natural category. For example, human face is represented in some feature space by a connected area of points (for different lighting conditions and face expressions), while all possible faces form much larger and more complex area that may be described using pdf, probability density function $P($ Face; $x)$ of different combinations of features that still resemble a face. In the Feature Space Model (FSM) a network of separable functions learns such pdfs from data [39].

Application of FSM to qualitative physics and to categorization problems showed that once expectations based on observation of partial behavior are internalized questions related to complex systems may be answered intuitively (that is without any explicit transformations). Problems involving many variables have to be solved by search, but search direction becomes almost unique when all constraints are intuitively taken into account. Mutual values of features are constrained, restricting possible solutions in such applications as discovery of scientific laws [31], [32]. Relations $A=f(B, C)$ between variables that are additive $A=$ $B+C$, inverse additive $A^{-1}=B^{-1}+C^{-1}$ or multiplicative $A=B \cdot C$ (as for example when resistances are added sequentially, in parallel, or when the Ohm's law $V=$ $I \cdot R$ is considered) show qualitatively identical behavior. For example, if the values of $B$ and $C$ increase then the value of $A$ should also increase, it cannot stay constant or decrease. Out of all 27 possible relations 13 are true and 14 false. This type of qualitative constraints are internalized and remembered, enabling predictions of qualitative behavior in complex cases. For $N$ variables out of $3^{N}$ possible solutions only a few will be in agreement with all constraints. For example, if $A_{1}=f\left(A_{2}, A_{3}\right) ; A_{2}=f\left(A_{3}, A_{4}\right) \ldots A_{N-2}=$ $f\left(A_{N-1}, A_{N}\right)$ the number of possible solutions that agree with all constraints is only $4 N+1$, and if some information is given solution may be unique. If it is known that $A_{1}$ and $A_{2}$ grows, then all other variables have to grow. In the brain such qualitative relation $A=f(B, C)$ may be implemented between 3 microcircuits, each representing one variable. Learning about the problem (for example, learning that $A$ and $B$ grows), activates some microcircuits, and this process activates all other microcircuits corresponding to variables that are uniquely defined, effectively completing the inference task without any reference to logic. This approach provides quite natural solution to problems discussed in [31], [32].

Analysis of a simple electric circuit using network that knows Ohm and Kirchoff laws [39] showed how such symbolic knowledge helps to find a solution to a problem that for most neural networks is very difficult to solve [44]. If the problem has several solutions a pair of variables that maximally constrain the third one should be selected first, leading to a systematic, but quite simple search similar to a typical reasoning: let's assume that $A$ and $B$, then what can be said about $C$, ignoring all other information. It is the activity of distributed network that decides whether relation $F\left(A_{1}, A_{2}, \ldots A_{N}\right)$ is feasible. The same mechanism is used to solve input-completion problems, where only some elements of the input vectors are defined. Setting some unknowns temporarily aside is equivalent to finding a projection of the whole situation on a lower-dimensional subspace of 
the known inputs. Once a point in this subspace is fixed, new dimension is added and unknown input values that are in agreement with all applicable constraints found (a one-dimensional line search procedure is sufficient). This geometric picture corresponds to successive enlargement of active subnetwork of resonating microcircuits in the brain.

In creative problem solving direct relations between variables, or object features, may be quite weak, because the neural circuits implementing these variables do not have direct connections, and thus probability of forming appropriate resonances may be very weak. The preparatory period introduces all relevant information simultaneously activating corresponding neural circuits that are mostly in the language areas of the dominant temporal lobe (presumably Superior Temporal Sulcus, STS), and that may recruit other circuits in the visual, auditory, somatosensory and motor areas. These circuits are now "primed" and may form many transient configurations. If the problem is easy, associations are quickly found and the results are not considered creative. If the problem is hard incubation period follows. This may be followed by an impasse and despair period, when inhibitory activity lowers activity of primed circuits allowing for recruitment of new circuits. Alternatively, after a longer period of distributed activity among primed circuits forming resonances between different neural assemblies, an interesting solution may arise for sufficiently long time to be amplified by emotional filters (emotional arousal may provide more neurotransmitters to the circuits involved). The third possibility, generating Aha! experience, is via indirect connections to the non-dominant (usually right) temporal lobe. Connections between left and right hemisphere require long projections and cannot carry precise information. Therefore right hemisphere has only a global view corresponding to a higher level of abstraction, or general categories. Distributed activation in the right hemisphere may forms various configurations that will activate back larger regions of the left hemisphere. Solution found via this route will usually be based on abstract ideas (common in mathematics). The result of the last two processes will probably be assessed as creative.

At the neural level creativity requires two components: distributed chaotic (fluctuating) neural activity that is responsible for imagination, and selection of interesting results. Imagination means that many transient patterns of excitations arise in parallel guided by the strength of neural connections. Most of these activations do not have much sense and are transient configurations, fleeting thoughts that escape the mind without being noticed. Selection is based on priming expectations, forming associations, arousing emotions, and in case of linguistic competence on phonological and semantic density around new words that find their way to the working memory, or pop up in mind.

Intelligence is correlated with creativity and it is quite likely that both have similar neurobiological basis. The Gfactor is highly correlated with working memory capacity, perceptual speed, choice and discrimination reaction times, the structure of event-related EEG potentials (ERP potentials), nerve conduction velocity, and cerebral glucose metabolic rate during cognitive activity [9]. Brains of creative people probably differ in the density of synaptic connections, contributing to the richer structure of the waveform of ERP potentials. This should also be true for the distal, interhemispheric connections. Denser neural connections may have an adverse efect on creating resonance states between closely associated concepts. In simpler brains such associations will dominate, making the responses more predictable, while in more complex brains there will be many competing resonances and thus larger repertoire of possible responses. Adding some neural noise may increase the chance to form resonance states if connections are weak, but will have little influence if connections are strong. This is a form of stochastic resonance phenomenon [45].

From this point of view results of experiments by Gruszka and Nȩcka [24] are not so puzzling. Less creative participants have weak connections even for close associations, therefore adding neural noise by showing them nonsensical priming words that appear very briefly before the second word of the pair is presented helps, while it will have much less influence on the creative people. On the other hand adding noise should help creative people to reach the resonance threshold if associations are distant, but for less creative people connections are simply too weak and priming may only lead to chaotic behavior. In another experimental condition [24] priming words were slightly misspelled version of the second word of the pair. For close associations this increases activity of the second word, and should always increase the chance of a resonance, reducing also latency of the response. However, for remote associations this will not help creative people because the two neural circuits need more energy (noise) in the intermediate circuits that may be activated only by nonsensical primes.

\section{CReativity at the lowest linguistic LeVel}

Perhaps the simplest activity in which creativity is frequently manifested is in understanding and creating new words. In languages with rich morphological and phonological compositionality (such as the Polish language) novel words appear even in normal conversation. Although these words are newly invented and cannot be found in any dictionary they may be understandable even without hearing them in a context. The simplest test for creative thinking in linguistic domain may be based on ingenuity of finding new names for products, web sites or companies that capture their characteristics. This has also practical value: attractive new names may be copyrighted and are always in demand.

The neuroscience of language in general, and word representation in the brain in particular, is far from being complete, but the cell assembly model of language has already strong experimental support [46],[47]. In this model words are represented as strongly linked subnetworks of microcircuits that bind articulatory and acoustic representation of spoken word, as well as it meaning. This is achieved by extending the phonological representation network to bind 
also related perceptions and actions, grounding its meaning in being-in-the-world. Various neuroimaging techniques confirm existence of such semantically extended networks. Psycholinguistic experiments show that acoustic speech input is quickly changed into categorical, phonological representation. A small set of phonemes (to be more precise, allophones correspond to actual resonances) is linked together in ordered string by a resonant state representing word form, and extended to include other microcircuits defining semantic concept. It has been conjectured (analyzing the N200 feature of ERPs) that phonological processing precedes semantic by about $90 \mathrm{~ms}$ [46].

To recognize a word in a conscious way activity of its subnetwork must win a competition for an access to the working memory [48]. Hearing a word activates string of phonemes increasing the activity (priming) of all candidate words and non-word combinations. Polysemic words probably have a single phonological representation that differs only by semantic extensions. Context priming selects extended subnetwork corresponding to a unique word meaning, while competition and inhibition in the winner-takes-all processes leaves only the most active candidate network. Similar patterns of brain activations for different word subnetworks will lead to strong transition probabilities between words and thus semantic and phonological associations.

The phonetically-detailed computational models of spoken word representation based on Adaptive Resonance Theory (ART) have been developed by Grossberg and his collaborators [49]-[51]. ART is essentially a general model of stable learning that includes bottom-up information filtering, horizontal competition between network nodes, and topdown attentional interactions between working memory and long-term memory. The ARTPHONE model [49] has been used for quantitative simulation of interesting phonological phenomena, such as phonemic restoration in noisy and silent conditions, while the ARTWORD model [50] can account for temporal chunking, word grouping, masking, rateindependent speech recognition and many other phenomena.

Suppose now that several keywords or a short text containing such keywords is given, priming the brain at the phonetic and semantic level. Experiments described below has been motivated by a real letter from a friend: "I am looking for a word that would capture the following qualities: portal to new worlds of imagination and creativity, a place where visitors embark on a journey discovering their inner selves, awakening the Peter Pan within. A place where we can travel through time and space (from the origin to the future and back), so, its about time, about space, infinite possibilities." The goal is thus to come up with a novel word that captures associations with a set of keywords. Large number of transient resonant configurations of neural cell assemblies may be formed in each second, exploring the space of all possibilities that agree with internalized constraints on the phonological structure of words (phonotactics of the language).

Autoassociative network based on modified correlation matrix memory [52] has been trained on a corpus of about

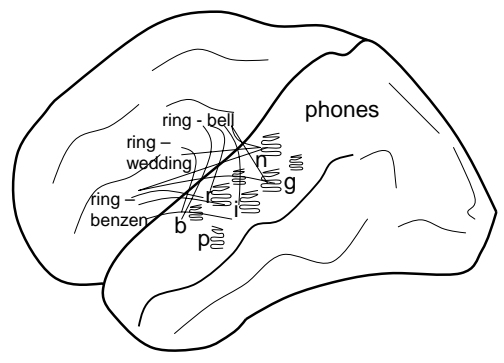

Fig. 1. Listening to a word activates phonetic resonances that form phonological representation, while context primes different extended subnetworks providing semantic representation.

1 million words to capture statistical correlations at the phonetic level. Unfortunately English spelling significantly differs from pronunciation, therefore such experiments should be done on phonologically encoded texts. In the simplest case the network is represented by a weight matrix:

$$
W_{i j}=\sum_{k} S_{k, i} S_{k, j}
$$

where $S_{k, i}$ is a binary vector indicating the presence of elementary word structure $i$ in the word $k$, called for brevity "a wordel". Ideally a wordel should represent an elementary resonance, activity of some microcircuit in the brain when the word is presented. In the simplest model wordels indicate presence of letters at selected positions. With 26 letters plus space each letter in temperature coding is replaced by a vector with 26 zeros, and a single 1 at the position of this letter in the alphabet. Thus 10-letter English words are represented by a string $S_{k}$ with 270 elements. After scanning a longer text the normalized weight matrix in such representation reflects probability of finding combinations of pairs of letters at some positions, with $W_{i j}$ far from diagonal approaching the product $p_{i} p_{j}$ of probabilities of finding letters $i, j$. Long-range correlations carry little information and therefore only blocks around diagonal of the matrix $\mathbf{W}$ differing on less than 3 positions (on the left and right) are used. More details may be captured using wordels based on bigrams. One could also introduce decomposition of words into morphemes, indicating presence of morphological components. The dimension of the weight matrix grows quickly when higher-order wordels are used.

Experiments with Polish text corpus and bigram representations have been conducted. With 35 letters in the Polish language the number of possible bigrams is 1225 , but actually less than 800 were found in texts. Restricting the number of letters in the word to 20 gives full matrices of dimension 16000. This is further reduced by the fact that correlations of elements differing on no more than two positions (neighbors and next-neighbors) were taken into account, making the number of $800 \times 800$ blocks equal to 94 , leaving about 60 million elements. Training autoassociative correlation memory allows for capturing statistical correlations between wordels. The world of possible word-forms 
constrained by these correlations may be explored by setting a threshold for probability of a word string (this should be normalized to account for different word length), and looping over all possible combinations of wordels. Using a low threshold this algorithm produces a huge stream of words, but increasing the threshold strings of quite interesting words are produced at a slower rate (most branches of the search tree lead to a dead end). Results from this experiment are not discussed further here because structural differences between Polish and English are too large.

Priming by keywords may be added as enhancement of probability of wordels appearing in keywords, changing probability of acceptance of novel strings. In the brain words that use larger subnetworks common to many words have higher chance to win competition, as they lead to stronger resonance states, with microcircuits that mutually support joint activity. This probably explains the tendency to use the same word in many meanings, and create many variants of words around the same morphemes. Creative brains have probably greater imagination, producing faster and more varied combinations, but also noticing interesting combination through emotional and associative filtering. Emotional filtering will be rather difficult to model, but in case of words filters based on phonological and semantic plausibility may be proposed. Phonological filters are essentially build in the autocorrelation matrix memory, but one could also construct some measures of phonological neighborhood density, counting the number of words that sound similar to a target word. Semantic neighborhood density measures should evaluate the number of words that have similar meaning to a target word, including similarity to morphemes that words may be decomposed to.

The algorithm that has been used for generating interesting English words contains the following steps:

1) Preparation: calculate probabilities for linking bigrams using a text corpus.

2) Read initial pool of keywords.

3) Find phonological and semantic associations with keywords to increase the pool (this may include foreign translations with different morphemes).

4) Create wordels: break all words into chains of phonemes and chains of morphemes.

5) Find all combinations of wordels.

6) Rank all candidate words according to their phonological probability, reject those below a given threshold (such as words that are difficult to pronounce).

7) Break each word into morphemes, and evaluate semantic density around it, providing the final ranking.

This algorithm gave surprisingly interesting results (details will be given in W. Duch and M. Pilichowski, in preparation). The following keywords from the email quoted above were extracted: portal, imagination, creativity, journey, discovery, travel, time, space, infinite. Some of the top words created include:

creativital, creatival; used by creatival.com creativery (creativity, discovery); used by creativery.com discoverity $=($ disc, disco, discover, verity, discovery, creativity, verity); used by some people

digventure =dig, digital, venture, adventure; new!

imativity (imagination, creativity); infinitime; new!

infinition (infinitive, imagination); a company name

journativity (journey, creativity); new!

learnativity; used by learnativity.com

portravel (portal, travel); used by portravel.com

sportal (space, sport, portal); used by sportal.com

timagination (time, imagination); company name

timativity (time, creativity); new!

tivery (time, discovery); new!

trime (travel, time); used frequently.

"Discoverity" has a connotation of discovering the truth (verity). "Digventure" is interesting because it is easy to pronounce, but also both "dig" and "venture" have many meanings and thus lead to many associations, creating a subnetwork of activity in the brain that resonates for a long time. In December 2005 Google did not found a single instance of this word, but a month later, after showing it in a presentation accessible via the Interent, this word appeared at an extremely long list of registered .com domains.

\section{DISCUSSION AND CONCLUSIONS}

Neurocognitive approach to creativity outlined in this paper may be implemented in many variants and at different level of neurobiological approximation, from detailed neural models to simple statistical approaches. From the methodological perspective simplest models should be tried first and their limitations clearly understood. The algorithm for creating new words based on the principles presented here, even in its drastically simplified form, produced many interesting words. Some of these words are novel, useful, appropriate and certainly fulfill all criteria for creative products. Applications of such algorithms include generation of names that can be sold and copyrighted, playing word games and understanding new words that have never been used before. It should be possible to compare words generated by such algorithms with words invented by humans. Computational creativity in this area may be easier to achieve than previously thought.

Same principles should apply to creativity in design, mathematics, and other domains, although in visual or abstract domain elementary transitions are not so easy to define as in the lexical domain. Imagination should be constrained by probabilities of composition of elementary operations; a guiding principle here is that these elementary operations are internalized as activations of specific brain subnetworks. Results of imagination should be filtered and ranked in a domain-specific way. In arts emotional reactions and beauty are rather difficult to formalize, but a network that learns individual preferences evaluating similarity to previous cases should be feasible. In abstract domains various measures of relevance or interestingness are possible.

Brain imaging and electrophysiological studies of brain activity during invention of new words and hearing of new words that require analysis would be quite interesting [46]. 
Probing associations and transition probabilities between brain states may be done using psychological experiments [24]. Research program on creativity that includes neuroscience, cognitive psychology and theoretical modeling, focused on word representation and creation, could be an entry to a detailed understanding of creativity, still considered to be one of the most mysterious brain processes. Results presented here support the idea that creative processes are based on ordinary cognitive processes. Research on creativity will hopfuly belong to the mainstream research areas in the near future.

\section{ACKNOWLEDGMENT}

Support by the Polish Ministry of Education and Science, research grant 2005-2007, is gratefully acknowledged.

\section{REFERENCES}

[1] R.J. Sternberg, Ed. Handbook of Human Creativity. Cambridge Uni. Press, 1998.

[2] M.L. Bink, R.L. Marsh, "Cognitive regularities in creative activity." Review of General Psychology, vol. 4, pp. 59-78, 2001.

[3] M.A. Boden, The Creative Mind: Myths and Mechanisms. London: Abacus, 1991.

[4] J.P. Guilford, The Nature of Human Intelligence. New York: McGrawHill 1967.

[5] R.J. Sternberg, "The theory of successful intelligence". Review of General Psychology, vol. 3, pp. 292-316, 1999.

[6] B. Sriraman, "The Characteristics of Mathematical Creativity". The Mathematics Educator, vol. 14(1), pp. 19-34, 2004.

[7] M.A. Runco, "Creativity". Annual Review of Psychology, vol. 55: pp 657-687, 2004.

[8] M. Runco and S. Pritzke, Eds. Encyclopedia of creativity, vol 1-2, Elsevier, 2005

[9] A. Jensen, "The g Factor: The Science of Mental Ability". Westport CT, Praeger, 1998.

[10] H. Gardner, Frames of Mind. The theory of multiple intelligences. New York: Basic Books, 1983.

[11] J. Duncan, R.J. Seitz, J. Kolodny, D. Bor, H. Herzog, A. Ahmed, F.N. Newell and H. Emslie, "A Neural Basis for General Intelligence". Science, vol 289(5478), pp. 457-460, 2000.

[12] R.J. Sternberg and J.E. Davidson, "Insight". In: [8]

[13] M. Jung-Beeman et al. "Neural Activity When People Solve Verbal Problems with Insight”. PLoS Biol 2(4): e111, 2004.

[14] E.M. Bowden, M. Jung-Beeman, J. Fleck, J. Kounios, "New approaches to demystifying insight". Trends in Cognitive Science, vol. 9, pp. 322-328, 2005.

[15] M. Sinclair, N.M. Ashkanasy, "Intuition: Myth or a Decision-making Tool?" Management Learning, Vol. 36, No. 3, 353-370 (2005)

[16] D. Shirley, J. Langan-Fox, "Intuition: A Review of the Literature". Psychological Reports, vol.79(2), pp.563-584, 1996.

[17] E. Policastro, "Intuition." Imagination, Cognition and Personality, vol. 20, Number 3 / 2000-2001, pp. 217 - 230

[18] J. Langan-Fox, D. Shirley, The Nature and Measurement of Intuition: Cognitive and Behavioral Interests, Personality, and Experiences. Creativity Research Journal vol. 15(3), pp. 207-222, 2003.

[19] M-H. Raidl and T.I. Lubart, "An empirical study of intuition and creativity". Imagination, Cognition and Personality, vol. 20(3), pp. 217-230, 2001.

[20] M.D. Lieberman, "Intuition: A social cognitive neuroscience approach". Psychological Bulletin, vol. 126, pp. 109-137, 2000.

[21] S. Dehaene, E. Spelke, P. Pinel, R. Stanescu, S. Tsivkin, "Sources of mathematical thinking: Behavioural and brain-imaging evidence". Science, vol. 284, 970-977, 1999.

[22] J. Rehling, Letter Spirit (Part Two): Modeling Creativity in a Visual Domain. PhD Thesis, Indiana University, 2001

[23] T.I. Lubart, "Models of the Creative Process: Past, Present and Future." Creativity Research Journal, vol. 13(4), pp. 295-308, 2001.

[24] A. Gruszka, E. Nęcka, "Priming and Acceptance of Close and Remote Associations by Creative and Less Creative People." Creativity Research Journal, vol. 14(2), pp. 193-205, 2002.
[25] S. Carson, J.B. Peterson and D.M. Higgins, "Latent inhibition and creative achievement in a high-achieving normative population". Journal of Personality and Social Psychology, vol. 85, 499-506, 2003.

[26] J. Goldenberg, D. Mazursky, S. Solomon, Science, vol 285(5433), pp. 1495-1496, 1999.

[27] J. Goldenberg, D. Mazursky, Creativity in Product Innovation, Cambridge Uni. Press, 2002.

[28] G. Henden, Intuition and its Role in Strategic Thinking. Norwegian School of Management, Dept. of Strategy and Logistics, Series of Dissertations, Oslo, April 2004.

[29] T.B. Ward, S.M. Smith, R.A. Finke, "Creative cognition". In: Sternberg, R.J (Eds), Handbook of Creativity, Cambridge Uni. Press, pp. 189-212, 1999.

[30] P. Langley, H.A. Simon, G.L. Bradshaw, and J.M. Żytkow, Scientific Discovery: Computational Exploration of the Creative Process. Cambridge, MA: MIT Press 1987.

[31] P. Langley, R. Jones, A computational model of scientific insight. In R. Sternberg (Ed.), The nature of creativity. Cambridge Uni. Press, 1988.

[32] S. Kocabas, P. Langley, An integrated framework for extended discovery in particle physics. Proc. 4th Int. Conf. on Discovery Science, Washington, D.C. Springer, pp. 182-195, 2001.

[33] J.R. Koza, M.A. Keane, M.J. Streeter, W. Mydlowec, J. Yu, G. Lanza, "Genetic Programming IV: Routine Human-Competitive Machine Intelligence". Kluwer Academic Publishers, 2003.

[34] M. Mitchell, "Analogy-Making as Perception: A Computer Model". Cambridge, MA: MIT Press, 1993.

[35] D.R. Hofstadter, "Fluid Concepts and Creative Analogies: Computer Models of the Fundamental Mechanisms of Thought". NY: Basic Books, 1995.

[36] W. Duch, R. Setiono, and J. Zurada. "Computational intelligence methods for understanding of data." Proceedings of the IEEE, 92(5):771805, 2004.

[37] W. Duch, M. Blachnik, "Fuzzy rule-based systems derived from similarity to prototypes." Lecture Notes in Computer Science, vol. 3316, 912-917, 2004.

[38] W. Duch, "Rules, Similarity, and Threshold Logic. Commentary on Emmanuel M. Pothos, The Rules versus Similarity distinction." Behavioral and Brain Sciences, vol. 28(1), pp. 23, 2005.

[39] W. Duch, G.H.F. Diercksen, "Feature Space Mapping as a universal adaptive system." Computer Physics Communications, vol. 87, pp. 341-371, 1995

[40] W. Duch, "Platonic model of mind as an approximation to neurodynamics." In: Brain-like computing and intelligent information systems, ed. S-i. Amari, N. Kasabov, Springer, Singapore, chap. 20, pp. 491$512,1997$.

[41] S. Grossberg, "The complementary brain: Unifying brain dynamics and modularity." Trends in Cognitive Sci, vol. 4, pp. 233-246, 2000.

[42] S. Edelman S, Intrator N, "Learning as extraction of low-dimensional representations." In: Medin D, Goldstone R, Schyns P (Eds.), Mechanism of Perceptual Learning. Academic Press, 1997.

[43] S. Edelman, "Neural spaces: a general framework for the understanding of cognition?" Behavioral and Brain Sci, vol. 24(4), 664-665, 2001.

[44] J.L. McClelland, D.E. Rumelhart, Parallel distributed processing. Cambridge, MA: MIT Press, 1987.

[45] T. Wellens, V. Shatokhin, A. Buchleitner, "Stochastic resonance." Reports on Progress in Physics, vol. 67, pp. 45-105, 2004.

[46] F. Pulvermüller, The Neuroscience of Language. On Brain Circuits of Words and Serial Order. Cambridge Uni. Press, 2003.

[47] F. Pulvermüller, Y. Shtyrov, R. Ilmoniemi, "Brain signatures of meaning access in action word recognition." Journal of Cognitive Neuroscience, vol. 17(6), pp. 884-892, 2005.

[48] W. Duch, "Brain-inspired conscious computing architecture." Journal of Mind and Behavior, vol. 26(1-2), 1-22, 2005.

[49] S. Grossberg, I. Boardman, C. Cohen, "Neural dynamics of variablerate speech categorization". J. of Experimental Psychology: Human Perception and Performance, vol. 23, 418-503, 1997.

[50] S. Grossberg, C.W. Myers, "The resonant dynamics of speech perception: Interword integration and duration-dependent backward effects." Psychological Review, vol. 107, pp. 735-767, 2000.

[51] S. Grossberg, "Resonant neural dynamics of speech perception". Journal of Phonetics, vol. 31, pp. 423-445, 2003.

[52] T. Kohonen, "Correlation matrix memories". IEEE Transactions on Computers C, vol 21, pp. 353-359, 1972. 